PROCEEDINGS OF THE

AMERICAN MATHEMATICAL SOCIETY

Volume 130, Number 6, Pages 1717-1723

S 0002-9939(01)06291-8

Article electronically published on October 17, 2001

\title{
INDEX OF B-FREDHOLM OPERATORS AND GENERALIZATION OF A WEYL THEOREM
}

\author{
M. BERKANI
}

(Communicated by Joseph A. Ball)

\begin{abstract}
The aim of this paper is to show that if $S$ and $T$ are commuting B-Fredholm operators acting on a Banach space $X$, then $S T$ is a B-Fredholm operator and $\operatorname{ind}(S T)=\operatorname{ind}(S)+i n d(T)$, where ind means the index. Moreover if $T$ is a B-Fredholm operator and $F$ is a finite rank operator, then $T+F$ is a B-Fredholm operator and $\operatorname{ind}(T+F)=\operatorname{ind}(T)$. We also show that if 0 is isolated in the spectrum of $T$, then $T$ is a B-Fredholm operator of index 0 if and only if $T$ is Drazin invertible. In the case of a normal bounded linear operator $T$ acting on a Hilbert space $H$, we obtain a generalization of a classical Weyl theorem.
\end{abstract}

\section{INTRODUCTION}

This paper is a continuation of our previous works [2], 3, 4, [5]. We consider a Banach space $X$ and $L(X)$ the Banach algebra of bounded linear operators acting on $X$. For $T \in L(X)$ we will denote by $N(T)$ the null space of $T$, by $\alpha(T)$ the nullity of $T$, by $R(T)$ the range of $T$ and by $\beta(T)$ its defect. If the range $R(T)$ of $T$ is closed and $\alpha(T)<\infty$ (resp. $\beta(T)<\infty)$, then $T$ is called an upper semi-Fredholm (resp. a lower semi-Fredholm) operator. A semi-Fredholm operator is an upper or a lower semi-Fredholm operator. If both $\alpha(T)$ and $\beta(T)$ are finite, then $T$ is called a Fredholm operator and the index of $T$ is defined by $\operatorname{ind}(T)=\alpha(T)-\beta(T)$.

Now for a bounded linear operator $T$ and for each integer $n$, define $T_{n}$ to be the restriction of $T$ to $R\left(T^{n}\right)$ viewed as a map from $R\left(T^{n}\right)$ into $R\left(T^{n}\right)$ (in particular $T_{0}=T$ ). If for some integer $n$ the range space $R\left(T^{n}\right)$ is closed and $T_{n}$ is a Fredholm (resp. semi-Fredholm) operator, then $T$ is called a B-Fredholm operator (resp. a semi-B-Fredholm) operator. In this case and from [2, Proposition 2.1] $T_{m}$ is a Fredholm operator and $\operatorname{ind}\left(T_{m}\right)=\operatorname{ind}\left(T_{n}\right)$ for each $m \geq n$. This enables us to define the index of a B-Fredholm operator $T$ as the index of the Fredholm operator $T_{n}$ where $n$ is any integer such that $R\left(T^{n}\right)$ is closed and $T_{n}$ is a Fredholm operator. Let $B F(X)$ be the class of all B-Fredholm operators. In [2] the author has studied this class of operators and has proved [2, Theorem 2.7] that an operator $T \in L(X)$ is a B-Fredholm operator if and only if $T=T_{0} \oplus T_{1}$, where $T_{0}$ is a Fredholm operator and $T_{1}$ is a nilpotent one.

The aim of this paper is to study the properties of the index of B-Fredholm operators and to derive a generalization of a classical Weyl theorem.

Received by the editors December 5, 2000

1991 Mathematics Subject Classification. Primary 47A53, 47A55.

(C)2001 American Mathematical Society 
It appears that the concept of Drazin invertibility plays an important role for the class of B-Fredholm operators. Let $A$ be an algebra with a unit. Following [14] we say that an element $x$ of $A$ is Drazin invertible of degree $k$ if there is an element $b$ of $A$ such that

$$
x^{k} b x=x^{k}, b x b=b, x b=b x .
$$

Recall that the concept of Drazin invertibility was originally considered by M. P. Drazin in [6] where elements satisfying relation (1) are called pseudo-invertible elements. The Drazin spectrum is defined by $\sigma_{D}(a)=\{\lambda \in C: a-\lambda I$ is not Drazin invertible $\}$ for every $a \in A$. In the case of a Banach algebra $A$ and from [5, Theorem 2.3 ] we know that the Drazin spectrum satisfies the spectral mapping theorem.

In the case of a bounded linear operator $T$ acting on a Banach space $X$, it is well known that $T$ is Drazin invertible if and only if it has a finite ascent and descent (Definition 2.1), which is also equivalent to the fact that $T=T_{0} \oplus T_{1}$, where $T_{0}$ is an invertible operator and $T_{1}$ is a nilpotent one. (See [14 Proposition 6] and [12, Corollary 2.2].) In [5, Theorem 3.4] we have shown that a bounded linear operator $T$ acting on a Banach space $X$ is a B-Fredholm operator if and only if its projection in the algebra $L(X) / F_{0}(X)$ is Drazin invertible, where $F_{0}(X)$ is the ideal of finite rank operators in the algebra $L(X)$. This characterization of B-Fredholm operators shows easily that the class of B-Fredholm operators is stable under finite rank perturbation and the product of two commuting B-Fredholm operators is a B-Fredholm operator [5, Corollary 3.5].

After giving some preliminaries in the second section, we prove in the third section that if $S, T$ are two commuting B-Fredholm operators, then the product $S T$ is a B-Fredholm operator and $\operatorname{ind}(S T)=\operatorname{ind}(S)+i n d(T)$. Moreover if $T$ is a B-Fredholm operator and $F$ is a finite rank operator, then $T+F$ is a B-Fredholm operator and $\operatorname{ind}(T+F)=\operatorname{ind}(T)$. Those two results give a positive answer to two open questions of [5]. We also show that if 0 is isolated in the spectrum of $T$, then $T$ is a B-Fredholm operator of index 0 if and only if $T$ is Drazin invertible. Then we define B-Weyl operators and the B-Weyl spectrum as follows:

Definition 1.1. Let $T \in L(X)$. Then $T$ is called a B-Weyl operator if it is a B-Fredholm operator of index 0 .

The B-Weyl spectrum $\sigma_{B W}(T)$ of $T$ is defined by $\sigma_{B W}(T)=\{\lambda \in \mathbf{C}: T-\lambda I$ is not a B-Weyl operator\}.

In Theorem 4.3 we show that for $T \in L(X)$ we have

$$
\sigma_{B W}(T)=\bigcap_{F \in F_{0}(X)} \sigma_{D}(T+F),
$$

and in the case a normal operator $T$ acting on a Hilbert space $H$, we show that

$$
\sigma_{B W}(T)=\sigma(T) \backslash E(T),
$$

where $E(T)$ is the set of isolated eigenvalues of $T$, which gives a generalization of the classical Weyl theorem. Recall that the classical Weyl theorem [15] asserts that if $T$ is a normal operator acting on a Hilbert space $H$, then the Weyl spectrum $\sigma_{W}(T)$ is exactly the set of all points in $\sigma(T)$ except the isolated eigenvalues of finite multiplicity, that is,

$$
\sigma_{W}(T)=\sigma(T) \backslash \Pi_{00}(T),
$$


where $\Pi_{00}(T)$ is the set of isolated eigenvalues of finite multiplicity and $\sigma_{W}(T)$ is the Weyl spectrum of $T$, that is, $\sigma_{W}(T)=\{\lambda \in \mathbf{C}$ such that $T-\lambda I$ is not a Fredholm operator of index 0$\}$. It is known from [ 8 , Theorem 6.5.2] that

$$
\sigma_{W}(T)=\bigcap_{F \in F_{0}(X)} \sigma(T+F) .
$$

Henceforth, if $M$ and $N$ are two vector spaces, the notation $M \simeq N$ will mean that $M$ and $N$ are isomorphic. We also define the infimum of the empty set to be $\infty$.

\section{Preliminaries}

Definition $2.1(3])$. Let $T \in L(X)$ and let $n \in \mathbf{N}$.

i) The sequence $\left(c_{n}(T)\right)$ is defined by $c_{n}(T)=\operatorname{dim} R\left(T^{n}\right) / R\left(T^{n+1}\right)$, and the descent of $T$ is defined by $\delta(T)=\inf \left\{n: c_{n}(T)=0\right\}=\inf \left\{n: R\left(T^{n}\right)=R\left(T^{n+1}\right)\right\}$.

ii) The sequence $\left(c_{n}^{\prime}(T)\right)$ is defined by $c_{n}^{\prime}(T)=\operatorname{dim} N\left(T^{n+1}\right) / N\left(T^{n}\right)$, and the ascent $a(T)$ of $T$ is defined by

$$
a(T)=\inf \left\{n: c_{n}^{\prime}(T)=0\right\}=\inf \left\{n: N\left(T^{n}\right)=N\left(T^{n+1}\right)\right\} .
$$

iii) The sequence $\left(k_{n}(T)\right)$ is defined by

$$
k_{n}(T)=\operatorname{dim}\left[\left(R\left(T^{n}\right) \cap N(T)\right) /\left(R\left(T^{n+1}\right) \cap N(T)\right)\right] .
$$

Definition $2.2([1])$. Let $T \in L(X)$ and let $\Delta(T)=\{n \in \mathbf{N}: \quad \forall m \in \mathbf{N} m \geq$ $\left.n \Rightarrow\left(R\left(T^{n}\right) \cap N(T)\right) \subset\left(R\left(T^{m}\right) \cap N(T)\right)\right\}$. Then the degree of stable iteration $\operatorname{dis}(T)$ of $T$ is defined as $\operatorname{dis}(T)=\inf \Delta(T)$.

Definition $2.3(7])$. Let $T \in L(X)$ and let $d \in \mathbf{N}$. Then $T$ has a uniform descent for $n \geq d$ if $R(T)+N\left(T^{n}\right)=R(T)+N\left(T^{d}\right)$ for all $n \geq d$, in other words, if $k_{n}(T)=0(n \geq d)$.

If in addition $R(T)+N\left(T^{d}\right)$ is closed, then $T$ is said to have a topological uniform descent for $n \geq d$.

Theorem 2.4 (7, Theorem 4.7]). Suppose that $T$ is a bounded operator with topological uniform descent for $n \geq d$ on the Banach space $X, n, d \in \mathbf{N}$, and that $V$ is a bounded operator that commutes with $T$. If $V-T$ is sufficiently small and invertible, then:

(a) $V$ has closed range and $k_{p}(V)=0$ for each integer $p \geq 0$.

(b) $c_{p}(V)=c_{d}(T)$ for each integer $p \geq 0$.

(c) $c_{p}^{\prime}(V)=c_{d}^{\prime}(T)$ for each integer $p \geq 0$.

Remark A. As it has already been observed in [2] it is immediately seen that a B-Fredholm operator is an operator of topological uniform descent. Using this fact and the properties of operators with topological uniform descent, we have the following properties of the index:

i) If $S, T \in B F(X), S T=T S$ and $\|T-S\|$ is small, then $\operatorname{ind}(T)=\operatorname{ind}(S)$.

(See [7, Theorem 4.6].)

ii) If $S, T \in B F(X), S T=T S$ and $T-S$ is compact, then $\operatorname{ind}(T)=\operatorname{ind}(S)$. (See [7, Theorem 5.8].)

iii) If $T \in B F(X), S T=T S,\|T-S\|$ is small and $T-S$ is invertible, then $S$ is a Fredholm operator and $\operatorname{ind}(S)=\operatorname{ind}(T)$. (See [7, Theorem 4.7].) In particular, if $T$ is a B-Fredholm operator and $n$ is an integer large enough, then $T-\frac{1}{n} I$ is a Fredholm operator and $\operatorname{ind}\left(T-\frac{1}{n} I\right)=\operatorname{ind}(T)$. 


\section{INDEX OF B-FREDHOLM OPERATORS}

If $T$ is a bounded linear operator $T$ such that both of $\alpha(T)$ and $\beta(T)$ are finite, then the range $R(T)$ of $T$ is closed and $T$ is a Fredholm operator. In the following theorem, we prove a similar result for B-Fredholm operators giving a simple characterization of this class of operators.

Theorem 3.1. Let $T \in L(X)$. Then $T$ is a B-Fredholm operator if and only if there exists an integer $n \in \mathbf{N}$ such that $\alpha\left(T_{n}\right)$ and $\beta\left(T_{n}\right)$ are finite.

Proof. Suppose that $T$ is a B-Fredholm operator and let $d=\operatorname{dis}(T)$. Then from [2] Proposition 2.6] we know that $R\left(T^{d}\right) \cap N(T)$ is of finite dimension and $R(T)+N\left(T^{d}\right)$ is of finite codimension. So $\alpha\left(T_{d}\right)$ and $\beta\left(T_{d}\right)$ are both finite.

Conversely suppose that $T \in L(X)$ and there exist $n \in \mathbf{N}$ such that $\alpha\left(T_{n}\right)$ and $\beta\left(T_{n}\right)$ are both finite. Then $R(T)+N\left(T^{n}\right)$ is of finite codimension and $N(T) \cap R\left(T^{n}\right)$ is of finite dimension. Since $N(T) \cap R\left(T^{n}\right)$ is of finite dimension, the sequence $\left(N(T) \cap R\left((T)^{p}\right)\right)_{p}$ is a stationary sequence for $p$ large enough. This shows that $d=\operatorname{dis}(T) \in \mathbf{N}, \quad R\left(T^{d}\right) \cap N(T)$ is of finite dimension and $R(T)+N\left(T^{d}\right)$ is of finite codimension. From [9] Lemma 3.1] we have $\frac{N\left(T^{d+1}\right)}{N\left(T^{d}\right)} \simeq N(T) \cap R\left(T^{d}\right)$, and from [9] Lemma 3.2] we have $\frac{R\left(T^{d}\right)}{R\left(T^{d+1}\right)} \simeq \frac{X}{R(T)+N\left(T^{d}\right)}$. It then follows that $c_{d}(T)<\infty$ and $c_{d}^{\prime}(T)<\infty$. Since the sequences $\left(c_{p}(T)\right)_{p}$ and $\left(c_{p}^{\prime}(T)\right)_{p}$ are stationary sequences then for $p \geq d$, we have $c_{p}(T)<\infty$ and $c_{p}^{\prime}(T)<\infty$. Moreover by [9 Lemma 3.1] we have $\frac{N\left(T^{2 p}\right)}{N\left(T^{p}\right)} \simeq N\left(T^{p}\right) \cap R\left(T^{p}\right)$, and from [9] Lemma 3.2] we have $\frac{R\left(T^{p}\right)}{R\left(T^{2 p}\right)} \simeq \frac{X}{R\left(T^{p}\right)+N\left(T^{p}\right)}$. Therefore $N\left(T^{p}\right) \cap R\left(T^{p}\right)$ is of finite dimension and $R\left(T^{p}\right)+N\left(T^{p}\right)$ is of finite codimension. In particular the two sets are closed. Using the Neubauer lemma [11] Proposition 2.1.1] it follows that $R\left(T^{p}\right)$ is closed. Hence $T_{d}$ is a Fredholm operator and so $T \in B F(X)$.

Theorem 3.2. Let $S, T$ be two commuting B-Fredholm operators. Then the product $S T$ is a B-Fredholm operator and ind $(S T)=\operatorname{ind}(S)+\operatorname{ind}(T)$.

Proof. From [5, Corollary 3.5] we know that $S T$ is a B-Fredholm operator. Moreover there exists an integer $N_{0}$ such that for any integer $n \geq N_{0}$, the operators $T-\frac{1}{n} I$ and $S-\frac{1}{n} I$ are both Fredholm operators, $\operatorname{ind}\left(T-\frac{1}{n} I\right)=\operatorname{ind}(T)$ and $\operatorname{ind}\left(S-\frac{1}{n} I\right)=\operatorname{ind}(S)$. Moreover for $n \geq N_{0}$ the difference $S T-\left(S-\frac{1}{n} I\right)\left(T-\frac{1}{n} I\right)=$ $\frac{1}{n}\left(S+T-\frac{1}{n} I\right)$ is of small norm if the integer $n$ is chosen large enough. Since $S T$ and $\left(S-\frac{1}{n} I\right)\left(T-\frac{1}{n} I\right)$ are B-Fredholm operators, then by the Remark A we have $\operatorname{ind}(S T)=\operatorname{ind}\left(\left(S-\frac{1}{n} I\right)\left(T-\frac{1}{n} I\right)\right)$. Since $S-\frac{1}{n} I$ and $T-\frac{1}{n} I$ are both Fredholm operators, then

$\operatorname{ind}\left(\left(S-\frac{1}{n} I\right)\left(T-\frac{1}{n} I\right)\right)=\operatorname{ind}\left(S-\frac{1}{n} I\right)+\operatorname{ind}\left(T-\frac{1}{n} I\right)$. Since $\operatorname{ind}\left(S-\frac{1}{n} I\right)=\operatorname{ind}(S)$ and $\operatorname{ind}\left(T-\frac{1}{n} I\right)=\operatorname{ind}(T)$, then $\operatorname{ind}(S T)=\operatorname{ind}(S)+\operatorname{ind}(T)$.

Proposition 3.3. Let $T \in L(X)$ be a B-Fredholm operator and let $F$ be a finite rank operator. Then $T+F$ is a B-Fredholm operator and $\operatorname{ind}(T+F)=\operatorname{ind}(T)$.

Proof. From [3, Corollary 3.10], it follows that $T+F$ is a B-Fredholm operator. Moreover there exists an integer $N_{0}$ such that for any integer $n \geq N_{0}, T-\frac{1}{n} I$ and $T+F-\frac{1}{n} I$ are Fredholm operators, $\operatorname{ind}\left(T-\frac{1}{n} I\right)=\operatorname{ind}(T)$ and $\operatorname{ind}\left(T+F-\frac{1}{n} I\right)=$ $\operatorname{ind}(T+F)$. Since $F$ is a finite rank operator and $T-\frac{1}{n} I$ is a Fredholm operator, by the usual properties of the index we have $\left.i n d\left(T+F-\frac{1}{n} I\right)\right)=\operatorname{ind}\left(T-\frac{1}{n} I\right)$. So $\operatorname{ind}(T+F)=\operatorname{ind}(T)$. 
Remark B. 1) If $K$ is a compact operator such that $R\left(K^{n}\right)$ is not closed for every positive integer $n$, then $K$ is not a B-Fredholm operator. So if $F$ is a finite rank operator, then $F$ is a B-Fredholm operator, but $K+F$ is not a B-Fredholm operator, otherwise $K=K+F-F$ would be a B-Fredholm operator. Hence the class of B-Fredholm operators is not stable under compact perturbation.

2) Let $T \in L(X)$. It is easily seen that $T$ is a B-Fredholm operator if only if $T^{*}$ is a B-Fredholm operator. Moreover in this case $\operatorname{ind}\left(T^{*}\right)=-\operatorname{ind}(T)$.

\section{B-Freholm operators of INDEX 0}

Lemma 4.1. Let $T \in L(X)$. Then $T$ is a B-Fredholm operator of index 0 if and only if $T=T_{0} \oplus T_{1}$, where $T_{0}$ is a Fredholm operator of index 0 and $T_{1}$ is a nilpotent operator.

Proof. If $T$ is a B-Fredholm operator of index 0 , then $X=X_{0} \oplus X_{1}$, where $X_{0}, X_{1}$ are closed subspaces of $X, T_{0}=T_{\mid X_{0}}$ is a Fredholm operator and $T_{1}=T_{\mid X_{1}}$ is a nilpotent operator. Moreover we have $\operatorname{ind}(T)=\operatorname{ind}\left(T_{n}\right)$ for $n$ large enough. Since $T_{1}$ is a nilpotent operator, then for $n$ large enough we have $R\left(T^{n}\right)=R\left(T_{0}^{n}\right)$ and $T_{n}=\left(T_{0}\right)_{n}$. From [2, Proposition 1] we have $\left.\operatorname{ind}\left(T_{0}\right)=\operatorname{ind}\left(\left(T_{0}\right)_{n}\right)\right)=\operatorname{ind}\left(T_{n}\right)=$ $\operatorname{ind}(T)=0$.

Conversely if $X=X_{0} \oplus X_{1}, T_{0}=T_{\mid X_{0}}$ is a Fredholm operator of index 0 and $T_{1}=T_{\mid X_{1}}$ is a nilpotent operator, then by the same arguments, $T$ is a B-Fredholm operator of index 0 .

Theorem 4.2. Let $T \in L(X)$ be such that 0 is isolated in the spectrum $\sigma(T)$ of $T$. Then $T$ is a B-Fredholm operator of index 0 if and only if $T$ is Drazin invertible.

Proof. If $T$ is a B-Fredholm operator of index 0 , then $X=X_{0} \oplus X_{1}$ such that $T_{0}=T_{\mid X_{0}}$ is a Fredholm operator of index 0 and $T_{1}=T_{\mid X_{1}}$ is a nilpotent operator. If $T_{0}$ is invertible, then $T$ is Drazin invertible. If $T_{0}$ is not invertible, as 0 is isolated in the spectrum of $T$, then it is also isolated in the spectrum of $T_{0}$. Since $T_{0}$ is a Fredholm operator of index 0 , it follows from [1, Proposition 2] that $T_{0}=T_{00} \oplus T_{01}$, where $T_{00}$ is invertible and $T_{01}$ is a nilpotent operator. So $T=T_{00} \oplus T_{01} \oplus T_{1}$, with $T_{00}$ invertible and $T_{01} \oplus T_{1}$ nilpotent. This shows that $T$ is Drazin invertible.

Conversely if $T$ is Drazin invertible, then $T$ is of finite ascent and descent. It follows from [12, Theorem 1.2] that there is an integer $p$ such that $a(T)=d(T)=p$ and $X=R\left(T^{p}\right) \oplus N\left(T^{p}\right)$. Let $X_{0}=R\left(T^{p}\right)$ and $X_{1}=N\left(T^{p}\right)$. Since $T_{0}=T_{\mid X_{0}}$ is an invertible operator and $T_{1}=T_{\mid X_{1}}$ is a nilpotent operator, from the precedent proposition it follows that $T$ is a B-Fredholm operator of index 0 .

Theorem 4.3. Let $T \in L(X)$. Then $\sigma_{B W}(T)=\bigcap_{F \in F_{0}(X)} \sigma_{D}(T+F)$.

Proof. Let $\lambda \notin \sigma_{B W}(T)$. Then $T-\lambda I$ is a B-Fredholm operator of index 0. From Lemma 4.1, we have $T-\lambda I=T_{0} \oplus T_{1}$, where $T_{0}$ is a Fredholm operator of index 0 and $T_{1}$ is a nilpotent operator. From [8, Theorem 6.5.2] there exists a finite rank operator $S_{0}$ such that $T_{0}+S_{0}$ is invertible. Set $S=S_{0} \oplus 0$; then $S$ is of finite rank operator and $(T-\lambda I)+S=T_{0}+S_{0} \oplus T_{1}$ is Drazin invertible. Hence $\lambda \notin \bigcap_{F \in F_{0}(X)} \sigma_{D}(T+F)$.

Conversely if $\lambda \notin \bigcap_{F \in F_{0}(X)} \sigma_{D}(T+F)$, then there is a finite rank operator $F$ such that $(T-\lambda I)+F$ is Drazin invertible. From Proposition 3.3, $(T-\lambda I)=$ $(T-\lambda I)+F-F$ is a B-Fredholm operator and $\operatorname{ind}(T-\lambda I)=\operatorname{ind}((T-\lambda I)+F)=0$, and $\lambda \notin \sigma_{B W}(T)$. 
From this theorem, we immediately obtain the following characterization of BWeyl operators.

Corollary 4.4. Let $T \in L(X)$. Then $T$ is a $B$-Weyl operator if and only if $T=$ $S+F$, where $S$ is Drazin invertible operator and $F$ is a finite rank operator.

It is known from [13, Theorem 7.7] that if $\lambda$ is isolated in the spectrum $\sigma(T)$ of a normal operator $T$ acting on a Hilbert space $H$, then $T-\lambda I$ is Drazin invertible. By the following theorem we give for such an operator, a generalization of a classical Weyl theorem [15].

Theorem 4.5. Let $T \in L(H)$ be a normal operator. Then $\sigma_{B W}(T)=\sigma(T) \backslash E(T)$, where $E(T)$ is the set of isolated eigenvalues of $T$.

Proof. If $\lambda \notin \sigma_{B W}(T)$ and $\lambda \in \sigma(T)$, then $T-\lambda I$ is a B-Fredholm operator of index 0 . Hence there exists an integer $n$ such that $R\left((T-\lambda I)^{n}\right)$ is closed. Since $(T-\lambda I)^{n}$ is a normal operator, then from [13. Theorem VI.3.6],

$$
H=R\left((T-\lambda I)^{n}\right) \oplus N\left((T-\lambda I)^{n}\right) .
$$

As $T-\lambda I$ is a normal operator, then from [13, Theorem VI.3.7], $N\left((T-\lambda I)^{n}\right)=$ $N((T-\lambda I))$. Hence $R((T-\lambda I))=R\left((T-\lambda I)^{n}\right)$ and $H=R((T-\lambda I)) \oplus N((T-\lambda I))$. Since $\lambda \in \sigma(T)$, then $N(T-\lambda I) \neq 0$. It follows that $\lambda$ is an isolated eigenvalue of $T$.

Conversely if $\lambda \in E(T)$, then from [10. Theorem 7.1] we have $X=X_{0} \oplus X_{1}$, where $X_{0}, X_{1}$ are closed subspaces of $X, T_{0}=(T-\lambda I)_{\mid X_{0}}$ is an invertible operator and $T_{1}=(T-\lambda I)_{\mid X_{1}}$ is a quasi-nilpotent operator. Since $T$ is a normal operator, then $T_{1}$ is also a normal operator. As $T_{1}$ is quasi-nilpotent, it is a nilpotent operator. Therefore $T-\lambda I$ is Drazin invertible. From Theorem 2.2 it is a B-Fredholm operator of index 0.

\section{REFERENCES}

[1] B. A. Barnes, Riesz Points and Weyl's Theorem. Integr. Equ. Oper. Theory 34 (1999) 187196. MR 2000d:47006

[2] M. Berkani, On a class of quasi-Fredholm operators. Integr. Equ. Oper. Theory 34 (1999), 244-249. MR 2000d:47023

[3] M. Berkani, Restriction of an operator to the range of its powers.. Studia Mathematica, Vol 140(2), (2000), 163-175. MR 2001g:47021

[4] M. Berkani, M. Sarih, On semi-B-Fredholm operators. To appear in Glasgow Mathematical Journal.

[5] M. Berkani, M. Sarih, An Atkinson-type theorem for B-Fredholm operators, To appear in Studia Mathematica.

[6] M. P. Drazin, Pseudoinverse in associative rings and semigroups. Amer. Math. Monthly 65 (1958), 506-514. MR 20:5217

[7] S. Grabiner, Uniform ascent and descent of bounded operators; J. Math. Soc. Japan 34, No. 2 (1982), 317-337. MR 84a:47003

[8] R. Harte, Invertibility and singularity for bounded linear operators; Marcel Dekker. New York, Basel, 1988. MR 90a:15019

[9] M. Kaashoek, Ascent, Descent, Nullity and Defect, a Note on a Paper by A.E. Taylor; Math. Annalen 172, 105-115 (1967). MR 36:5719

[10] J. J. Koliha, A generalized Drazin inverse, Glasgow Math. J. 38 (1996), 367-381. MR 98b:46065

[11] J. P. Labrousse, Les opérateurs quasi-Fredholm: une généralisation des opérateurs semiFredholm; Rend. Circ. Math. Palermo (2), 29 (1980), 161-258. MR 83c:47022

[12] D. C. Lay, Spectral analysis using ascent, descent, nullity and defect; Math. Ann. 184, 197-214 (1970). 
[13] D. C. Lay, A. E. Taylor, Introduction to Functional Analysis, Second edition, Wiley, New York, 1980. MR 81b:46001

[14] S. Roch, B. Silbermann, Continuity of generalized inverses in Banach algebras. Studia Mathematica 136 (3), (1999), 197-227. MR 2000m:46101

[15] H. Weyl, Über beschränkte quadratische Formen, deren Differenz vollstetig ist. Rend. Circ. Mat. Palermo 27 (1909), 373-392.

Département de Mathématiques, Faculté des Sciences, Université Mohammed I, OuJDA, MAROC

E-mail address: berkani@sciences.univ-oujda.ac.ma 